\title{
Numerical calculation of the frequency response of the radiation coupling in lossy medium
}

\author{
M. G. S. Ali and A. Raouf Mohamed \\ Physics Department, Faculty of Science, Minia University, Egypt
}

(Received 9 November 1996)

\begin{abstract}
A numerical form for the radiation coupling between two co-axial disks separated by a medium exhibiting acoustic absorption shall be presented. This method has been used to show that diffraction and absorption can be treated as two separable processes. The radiation-coupling gain is applicable to the correction of the experimental data. The results are compared with published theoretical data.
\end{abstract}

Keywords : Diffraction, Absorption, Convolution, Radiation coupling

PACS number: 43. 40. Le

\section{INTRODUCTION}

The solution of the transient radiation coupling between two co-axial disks considered so far are only applicable for an ideal coupling medium which exhibits neither absorption nor dispersion. The measurement of the absorption or the velocity of ultrasound in a medium becomes complicated when we consider the diffraction occurs at the transmitting and receiving transducers of finite dimension. ${ }^{1)}$ Kozina and Makarov, ${ }^{2}{ }^{2}$ considered a basic diffraction phenomena in the time domain which showed that, a disturbance measured at a point in the field of a baffled plane piston radiator considered of two parts: a direct wave from the whole transducer face and a secondary disturbance, known as the edge wave, emanating from a line source around the periphery of the radiator. Several workers $^{3-5)}$ have explained diffraction corrections for measurement of ultrasonic velocity and absorption from a theoretical point of view. Khmunin ${ }^{6,7)}$ introduced the influence of intrinsic attenuation in the medium, but he neglected the effect of the resulting change in the transfer function upon measurements of velocity or attenuation. In case of two disks of same diameters and co-axially aligned,
Rhyne $^{8)}$ has found an exact solution of the total force on the receiver due to the velocity impulse from the transmitter.

In this paper a numerical approach is presented to extend Rhyne ${ }^{8)}$ solutions of the radiation coupling process from coupling in an ideal medium to that in an absorbing medium. The solution of the radiation coupling transfer function is in the form of Laplace domain representation, which can be readily related to the wave processes occurring within the medium spectra modelled. The technique shall be used to study the system such that, the diffraction, and absorption in the coupling medium are separate factors.

\section{THEORY}

For two disks of the same diameter co-axially aligned in ideal medium, Rhyne has found an exact solution to the total force on the receiver due to a velocity impulse from the transmitter. The medium between the transmitter and receiver is isotropic and homogenous and supports only compressional waves. Rhyne used the Stepanishen potential impulse response solution ${ }^{9)}$ to evaluate the surface integral of the velocity potential over the receiver face which is 


$$
\bar{H}(z, a, t) \begin{cases}=0 & t<t_{1} \\ =2 u a^{2} \cos ^{-1}\left\{\frac{\tau}{2 a}\right\} & \\ -\frac{u^{3}}{2} \sqrt{\left(t^{2}-t_{1}^{2}\right)\left(t_{2}{ }^{2}-t^{2}\right)} & t_{1} \leq t \leq t_{2} \\ =0 & t_{2}<t\end{cases}
$$

where $t_{1}=z / u, t_{2}=\sqrt{\left(4 a^{2}+z^{2}\right) / u_{2}}, \tau=\sqrt{(u t)^{2}-z^{2}}, u$ is the wave propagation velocity, $a$ is the transducer radius and $z$ the distance between the transm tter and receiver. The force impulse response at the receiver due to a velocity impulse disturbance from the transmitter is given by differentiating Eq. (1) with respect to time. Multiplying by medium density and then dividing by the real acoustic impedance on the transmitter will give Rhyne's result for the disk force coupling impulse response,

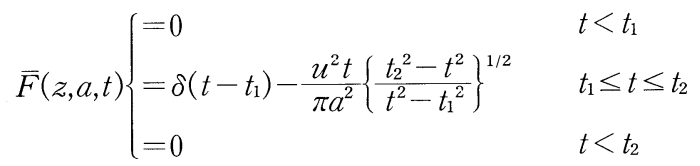

Furthermore, Rhyne found the corresponding transfer function in analytical form by using Fourier transformation of Eq. (2). The solution takes the form of a series expansion, which converges extremely rapidly. The first three terms in this solution are sufficient for the range of considered geometries. Therefore the solution becomes:

$$
\begin{aligned}
F(z, a, \omega)= & \cos \left(\omega t_{3}\right)+J \sin \left(\omega t_{3}\right)-\frac{u^{2} t_{4} t_{3}}{a^{2}}\left\{\frac{t_{4}+t_{2}}{t_{4}+t_{1}}\right\}^{1 / 2} \\
& \cdot\left\{J_{0}\left(\omega t_{3}\right)+j J_{1}\left(\omega t_{3}\right)\right\}+\frac{u^{2} t_{3}}{a^{2}}\left\{\frac{t_{4}+t_{1}}{t_{4}+t_{1}}\right\}^{1 / 2} \\
& \cdot\left[1-\frac{t_{4}}{2\left(t_{4}+t_{1}\right)}+\frac{t_{4}}{2\left(t_{4}+t_{2}\right)}\right] \\
& \cdot\left[J_{0}\left(\omega t_{3}\right)-\frac{J_{1}\left(\omega t_{3}\right)}{\omega t_{3}}+j J_{1}\left(\omega t_{3}\right)\right]
\end{aligned}
$$

where $J_{0}$ and $J$ are Bessel functions of the first kind, $t_{3}=\left(t_{2}-t_{1}\right) / 2$ and $t_{4}=\left(t_{2}+t_{1}\right) / 2$.

\section{RADIATION COUPLING IN ABSORBING MEDIUM}

The solution to the transient radiation coupling between two co-axial considered so far are only applicable for an ideal coupling medium which exhibits neither absorption nor dispersion. Because diffraction effects result from interference between wavelets from different sources arriving at the same field point, then all the changes at the wavelets brought about by the supporting medium during propagation will alter the interference pattern. The effect of a non-ideal medium upon the form of the radiation coupling solutions is of interest regarding the manner in which diffraction corrections must be made to the measured data. In particular, it is important to know to what extent radiation coupling in an absorbing medium can be considered as a diffaction process in conjunction with plane wave acoustic absorption and considering them as a separate factors.

In case of an ideal medium, the radiation coupling impulse response can be interpreted in the following manner. The disturbance to the transmitter face is a Dirac impulse of velocity, which by definition has infinite amplitude and zero duration such that its time integral is unity. The total force acting on the receiver face at any moment in time, $t$, is therefore due to waves arriving at the receiver which have propagated along a path length $(u t)$. The force coupling impulse responses such as the one in Eq. (2) gives a net signal weighting and a delay for every path length coupling the transmitter to the receiver. The effect of acoustic absorption on the supporting medium produces appears as delay on the net signal weight.

The response of the radiation coupling filter of an absorptive medium can be written as

$$
F(z, a, u, t)=F(z, a, t) * \exp \left(-\alpha_{\omega} u t\right)
$$

Where $*$ denotes the convolution operator, $\alpha_{\omega}$ is the absorption coefficient of the medium at frequency $\omega$ and $u t$ gives the path length in which the waves must have propagated along before arriving at the receiver.

The transfer function of the radiation-coupling filter for an absorptive medium is therefore given by

$$
F\left(z, a, \alpha_{\omega}, u, \omega\right)=\int_{-\infty}^{+\infty} F(z, a, u, t) \exp (-j \omega t) d t
$$

Equation (5) is the Fourier transform integral of impulse response function enclosed in Eq. (4). Equation (5) can be evaluated numerically for each frequency value, $\omega$, at which the transfer function is to be evaluated. A radiation coupling impulse response must be formed assuming that the coupling medium is ideal with a propagation velocity $u$.

The real frequency model is obtained from the impulse responses by Fourier transformation and replacing the time domain convolution by fiequency domain multiplication. 


\section{G. S. ALI and A. R. MOHAMED : FREQUENCY RESPONSE OF RADIATION COUPLING}

\section{RESULTS}

Calculations considered here are most conveniently and rapidly performed by ensuring that the frequency values at which the transfer function is evaluated corresponding to those using a fast Fourier transform (FFT) algorithm. The simulation was applied using disk of radius $7.5 \mathrm{~mm}$. Figure 1 shows the transfer function in Eq. (3) as a function of frequency at fixed diameter and distance. Figure 2 shows the radiation coupling in ideal medium with different values of the distance between the transmitter and receiver. It is clear from these figures that the radiation coupling filtering process exhibits a high-pass filter and approximately afler 1 $\mathrm{MHz}$ the gain of the radiation coupling filter asymptotically approaches constant value with some

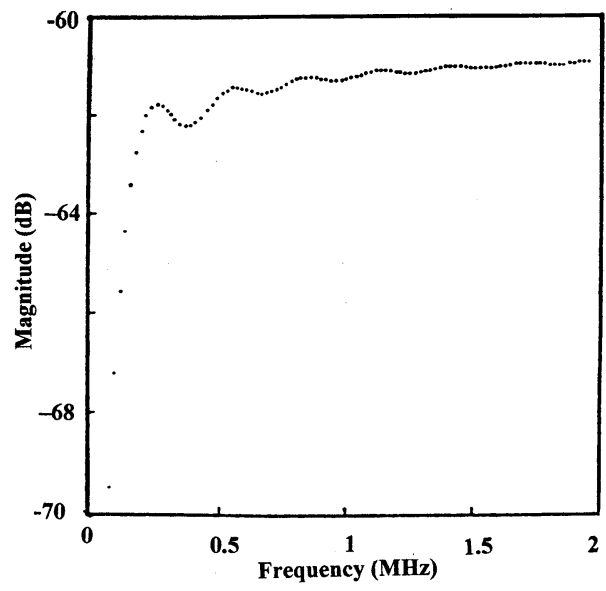

Fig. 1 Radiation coupling transfer function in ideal medium as a function of frequency (distance $=30 \mathrm{~mm}$, radius $=7.5 \mathrm{~mm}$ ).

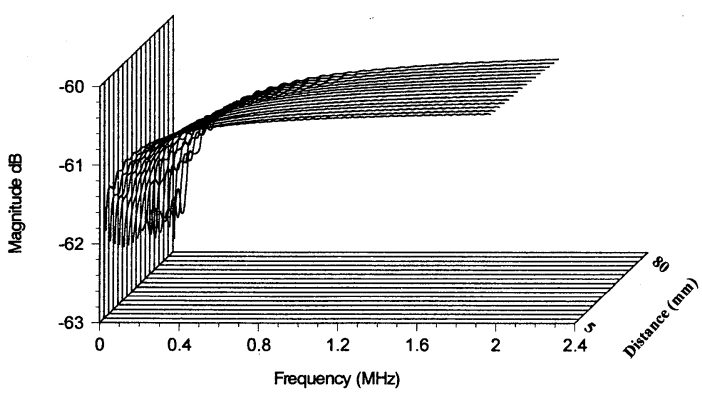

Fig. 2 Radiation coupling transfer function with different values of distances between the transmitter and receiver (radius $=7.5 \mathrm{~mm}$ ). ripple. The effect of the medium absorption on the transfer function is shown in Fig. 3 with different values of absorption coefficient, where curve (a) shows the classical absorption with $\alpha / f^{2}=1 \times 10^{-11}$ $\mathrm{np} / \mathrm{m}$ and curve (b) the absorption coefficient equal to five times that in curve (a). From this figure it can be seen that the transfer function damped with increasing frequency because the attenuation is strongly dependent on frequency. The transfer function as a function of distance at fixed diameter and frequency can also be calculated by Eq. (5).

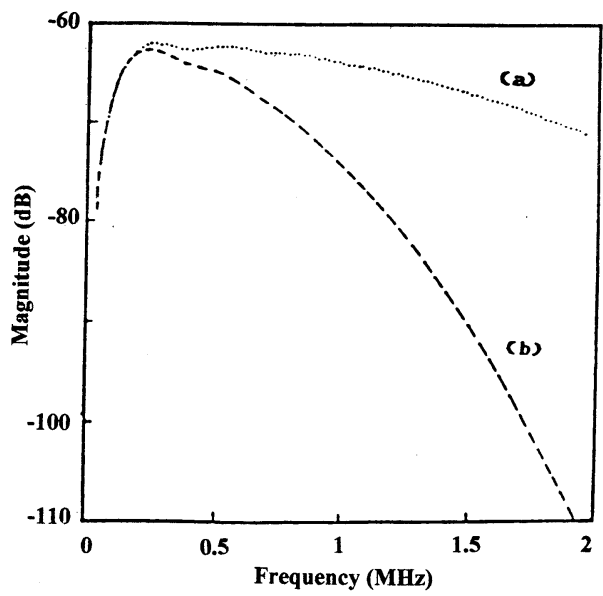

Fig. 3 Radiation coupling transfer function in absorbing medium as a function of frequency (distance $=30 \mathrm{~mm}$, radius $=7.5 \mathrm{~mm}$ ). (a) $\alpha /$ $f^{2}=1 \times 10^{-11} \mathrm{np} / \mathrm{m}$. (b) $\alpha / f^{2}=5 \times 10^{-11} \mathrm{np} /$ $\mathrm{m}$.

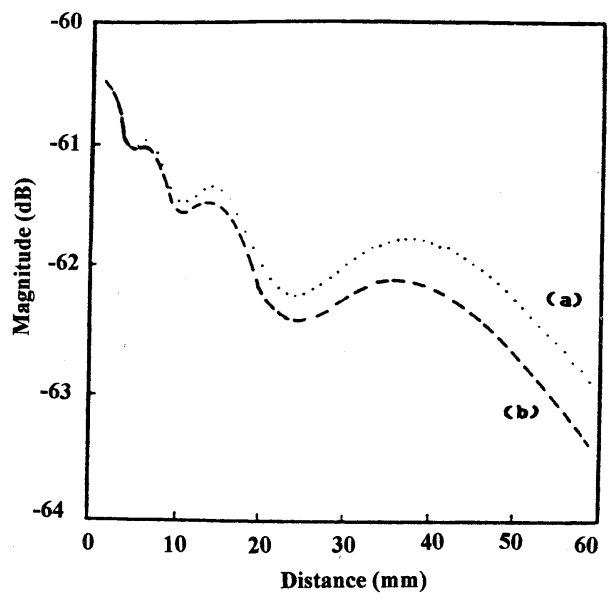

Fig. 4 Radiation coupling transfer function at $300 \mathrm{kHz}$ with variable distance (a) for ideal medium (b) for $\alpha=0.9 \mathrm{np} / \mathrm{m}$. 
The transfer function for each distance separation can be calculated to select the amplitude at some fixed frequency. Figure 4 shows the transfer function as a function of separation at frequency 300 $\mathrm{kHz}$ and propagation velocity $1,000 \mathrm{~ms}^{-1}$ afier the correction of the plane wave intrinsic absorption in the medium $(\alpha=0.9 \mathrm{np} / \mathrm{m})$. The same procedure has been selected in Fig. 5 but with absorption coefficient five times that in Fig. 4. The results obtained in Figs. 4 and 5 agreed in principle with Bacon $^{10)}$ who used a discrete element model. The advantage of the numerical solution represented by Eq. (5) over the discrete model used by Bacon ${ }^{10)}$ is seen in the computation time consumed to produce the results. The consumed time was $1 \mathrm{hr}$ and 20 mins in Bacon model and $50 \mathrm{~min}$ in the new model using the same computer system.

In order ton investigate the absorption effect on radiation coupling transfer function performance the simulation was applied to a medium of soft biological tissue with an absorption coefficient of 1 $\mathrm{dB} \mathrm{MHz} \mathrm{MH}^{-1} \mathrm{~cm}^{-1}$ Figure 6 shows the transfer functions with different values of distance between the transmitter and receiver. It is clear from this figure that the magnitude of the transfer function is strongly dependent on the filtering effects of absorption and depth.

For a given transducer geometry, the radiation coupling transfer function in such a medium is then calculated using the numerical method based upon Eq. (5). The resultant spectrum is analyzed first by

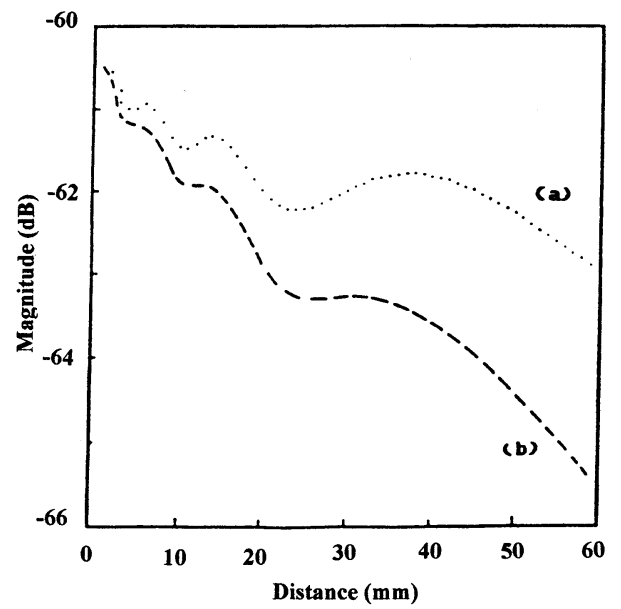

Fig. 5 Radiation coupling transfer function at $300 \mathrm{kHz}$ with variable distance (a) for ideal medium (b) for $\alpha=4.5 \mathrm{np} / \mathrm{m}$.

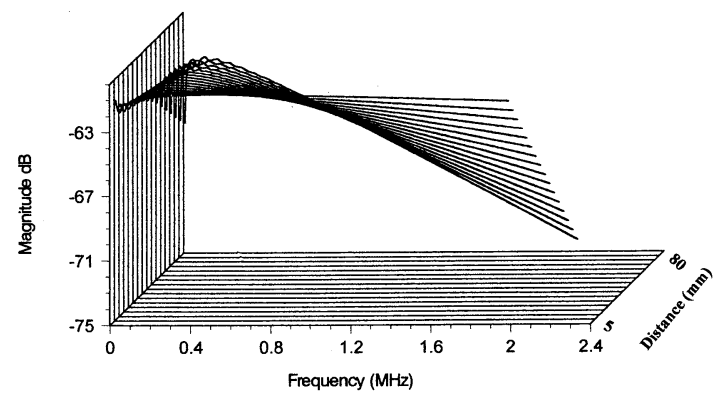

Fig. 6 Radiation coupling transfer function in soft biological tissue medium with different number of distance between the transmitter and receiver.

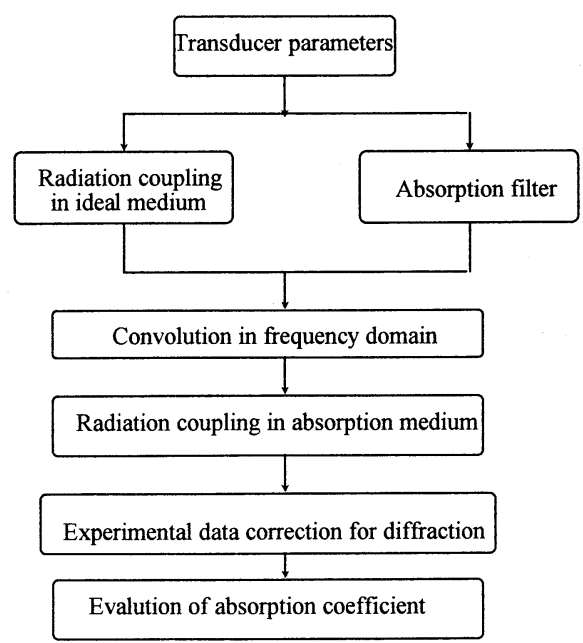

Fig. 7 Block diagram of the procedure to evaluate the ultrasonic absorption in the test medium.

correcting for diffraction using Eq. (3) and then calculating the absorption coefficient as a function of frequency. Finally. the value of the absorption coefficient calculated can be compared with the defined value to give the error as a function of frequency. Figure 7 represents a block diagram for the selected procedure to evaluate the absorption coefficient of the test medium.

\section{CONCLUSION}

The well-known analytical solution for the radiation coupling impulse response between two coaxial disks of the same size in an ideal medium has been extended to that in an absorbing medium. A 


\section{G. S. ALI and A. R. MOHAMED : FREQUENCY RESPONSE OF RADIATION COUPLING}

numerical approach for the radiation coupling transfer function between two disks separated by an absorbing medium has been developed. The diffraction and plane wave absorption in the acoustic field are treated as separable processes. Furthermore, the algorithm can be used effectively in real data for correction to get the final values of absorption and velocity.

\section{REFERENCES}

1) H. Seki, A. Granato and R. Truell, "Diffraction effects in the ultrasonic field of a piston source and their importance in the accurate measurement of attenuation," J. Acoust. Soc. Am. 28, 230 (1956).

2) O. G. Kozina and G. I. Makarov, "Transient processes in the acoustic field generated by a piston membrane of arbitrary shape," Sov. Phys. Acoust. 7(1), 39 (1961).

3) M. B. Gitis and A. S. Khimunin, "Diffraction effects in ultrasonic measurements (Review)," Sov. Phys.
Acoust. 14, 413 (1969).

4) K. Yamada and Y. Fujii, "Acoustic response of a circular receiver to a circular source of different radius," J. Acoust. Soc. Am. 40, 1193 (1966).

5) E. P. Papadakis, "Effect of input amplitude profile upon diffraction loss and phase change in a pulseecho system," J. Acoust. Soc. Am. 49, 166 (1971).

6) A. S. Khimunin, "Numerical calculation of the diffraction corrections for the precise measurement of ultrasonic absorption," 27, 173 (1975).

7) A. S. Khimunin, "Numerical calculation of the diffraction corrections for the precise measurement of ultrasonic phase velocity," Acustica 32, 192 (1975).

8) T. L. Rhyne, "Radiation coupling of a disk to a plane and back or a disk to a disk: an exact solution," J. Acoust. Soc. Am. 61, 318 (1977).

9) R. Stepanishen, "Transient radiation from pistons in an infinite planar baffle," J. Acoust. Soc. Am. 49, 1629 (1971).

10) R. A. Bacon and R. C. Chirers, "Radiation coupling of a disc to a disc : A numerical approach," Acoust. Lett. 5, 22 (1981). 